\title{
Diffusion-based Regularisation Strategies for Variational Level Set Segmentation
}

\author{
Maximilian Baust \\ baust@in.tum.de \\ Darko Zikic \\ zikic@cs.tum.edu \\ Nassir Navab \\ navab@cs.tum.edu
}

Computer Aided Medical Procedures

Technische Universität München

Munich, GER

\begin{abstract}
Variational level set methods are formulated as energy minimisation problems, which are often solved by gradient-based optimisation methods, such as gradient descent. Unfortunately, the gradient obtained by applying the calculus of variations is not suitable, because it is only an element of the function space $L^{2}$ making it prone to lead into wrong local minima. Consequently, some regularisation strategy - be it the restriction to signed distance functions or the choice of smooth function spaces - is necessary. In this paper we propose diffusion-based regularisation strategies and compare them to the recently proposed ones of Charpiat et al. and Sundaramoorthi et al. From this comparison we derive two general regularisation paradigms at the level of update equations and show that the diffusion-based paradigm enjoys both theoretical and practical advantages, such as an improved convergence rate, while being of the same computational complexity as the other paradigm.
\end{abstract}

\section{Introduction}

Many variational level set methods for image segmentation can be summarized in the following recipe. First, design an energy $E$ which gets minimized by the optimal configuration of the embedding function $\phi$ :

$$
\min _{\phi \in V} E(\phi) .
$$

Second, apply the calculus of variations to obtain $\nabla E(\phi)$ :

$$
\left.\frac{d}{d s} E(\phi+s \psi)\right|_{s=0}=\int_{\Omega} \nabla E(\phi) \cdot \psi d x=\langle\nabla E(\phi), \psi\rangle_{L^{2}}=0 .
$$

Third, solve (1) via gradient descent, which leads to the continuous evolution equation

$$
\partial_{t} \phi=-\nabla E(\phi)
$$

and the discrete update equation

$$
\phi^{t+\tau}=\phi^{t}-\tau \nabla E\left(\phi^{t}\right) .
$$


As already indicated in (2), the recipe described above implicitly assumes that $V=L^{2}$. Therefore it is mathematically correct to refer to $\nabla E$ as the $L^{2}$-gradient and not simply the gradient. Unfortunately, the $L^{2}$-gradient is, to put it simply, too local and therefore prone to lead into an undesired local minimum (see [ $\mathbf{\theta}]$ or [ $[\mathbf{U}]$ for further details). Thus regularisation strategies are necessary to avoid these undesired local minima and one can classify them as either implicit or explicit.

\subsection{Implicit Regularisation (Choosing a Smooth Subspace of $V$ )}

The oldest example for implicit regularisation is most probably the restriction of $\phi$ to the space of signed distance functions. However, the term "space" is not correctly used here, because signed distance functions do not form a function space in the mathematical sense as the sum of two signed distance functions is not a signed distance function for instance. Even if this would be the case, the updates $\nabla E\left(\phi^{t}\right)$ are also no signed distance functions and thus periodical re-initialisation is necessary (see [ $\square]$ for instance).

Another implicit regularisation strategy can be seen in the usage of parametrisations leading to function spaces spanned by smooth basis functions such as radial basis functions, which was done by Ho et al. [曰], Morse et al. [四], Slabaugh et al. [四], and Benoit et al. [प]. Approaches using splines, such as the MetaMorphs framework of Huang et al. [四] or the variational B-spline framework of Bernard et al. [ [ ] are also related to this strategy.

Recently, Bresson et al. employed the space of functions of bounded variation $B V$ in [ $[$ ]. However, their approach is restricted to a special functional $E$. Another, more general, choice was suggested by Charpiat et al. [ [ ] as well as Sundaramoorthi et al. [四], who restricted $V$ to the space of infinitely many times continuously differentiable functions $C^{\infty}$ or spaces of weakly differentiable functions, such as the Sobolev space $H^{1}$. Two years later, $C^{\infty}$ was also used by Bar and Sapiro in [ $\square]$, but based on a slightly different mathematical justification. We will see in Sec. 4 that, if we use a gradient descent to minimize $E$, the restriction to the function spaces suggested in $[\square, \boldsymbol{Q}, \boldsymbol{⿴ 囗 十}]$ results in an update equation of the form

$$
\phi^{t+\tau}=\phi^{t}-\tau \mathscr{R}\left[\nabla E\left(\phi^{t}\right)\right],
$$

where $\mathscr{R}[\cdot]$ is a certain regularisation operator.

It deserves credit, that the drawbacks of the $L^{2}$-gradient as well as sophisticated implicit regularisation strategies have been discovered much earlier in the registration community (e.g. Trouvé in 1998 [四]) than in the segmentation community.

\subsection{Explicit Regularisation (Regularising the Energy E)}

In contrast to implicit regularisation strategies, explicit strategies aim at regularising the energy $E$ directly:

$$
\min _{\phi \in L^{2}} E(\phi)+\lambda R(\phi)
$$

where $R$ is an additional regularisation term, with $\lambda>0$ controlling its influence. The first approach in this context is the one of Li et al. [ $\square]$, who suggested the regulariser

$$
R(\phi)=\int_{\Omega}\left(|\nabla \phi|^{2}-1\right)^{2} d x
$$


enforcing locally the shape of a signed distance function. This signed distance regularisation results in an evolution equation that includes

$$
\nabla R(\phi)=-\operatorname{div}\left(\left(1-\frac{1}{|\nabla \phi|}\right) \nabla \phi\right) .
$$

Unfortunately, the non-linearity of (8) does not allow the corresponding finite difference approximation to be written as a matrix vector multiplication and thus the applicability of efficient numerical schemes, such as semi-implicit time discretisations. The same holds true for a curvature-based regularisation, such as adding $\nabla R(\phi)=-\operatorname{div}(\nabla \phi /|\nabla \phi|)$ to the evolution equation, because the curvature operator is also non-linear.

\subsection{Regularisation Strategies based on Diffusion}

Similar to []] we focus on explicit regularisation strategies. However, we focus on regularisers of the form

$$
R(\phi)=\int_{\Omega}(\nabla \phi)^{T} g \nabla \phi d x,
$$

where the weight function $g$ solely depends on the image function $I$ and not on $\phi$ causing the resulting gradient

$$
\nabla R(\phi)=-\operatorname{div}(g \nabla \phi)
$$

to be linear with respect to $\phi$. Thanks to the linearity of (10) the resulting update equation can be written in the form

$$
\phi^{t+\tau}=\mathscr{R}\left[\phi^{t}-\tau \nabla E\left(\phi^{t}\right)\right],
$$

which will be shown in Sec. 3. It is obvious that, if the same regulariser is used, (5) and (11) are of the same computational complexity, but we will see that (11) has some desirable advantages which cannot be achieved by (5).

We want to emphasize at this point that a diffusion regularization has already been used in the well-known Mumford-Shah model [미] and its favourable theoretical as well as numerical properties concerning this model have also been proven, see [ $\square$ ] for instance. The focus of this paper is to introduce it from a general point of view and to relate it to the implicit regularization strategies of Charpiat et al. [回] and Sundaramoorthi et al. [四].

\section{Why Diffusion Regularisation}

Introducing a diffusion regularisation has theoretical as well as practical implications, which both need to be discussed.

\subsection{Theoretical Considerations}

A necessary theoretical assumption is the ellipticity condition

$$
g \geq c
$$

for some $c>0$. Provided (12) holds, the resulting evolution equation

$$
\partial_{t} \phi=-\nabla E(\phi)+\lambda \operatorname{div}(g \nabla \phi)
$$




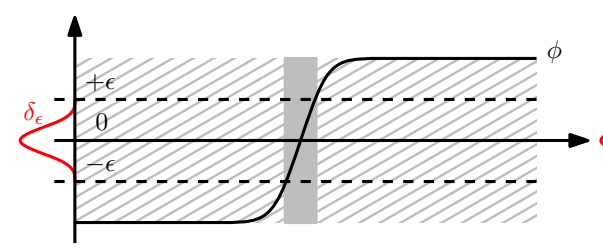

(a)

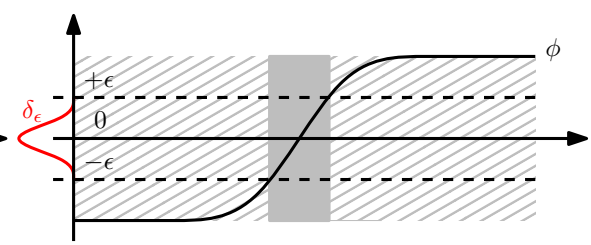

(b)

Figure 1: The smeared-out Dirac distribution $\delta_{\varepsilon}$ divides the image domain into an diffusiondominated area (grey stripes) and a competitive area (grey). When $\phi$ becomes flatter the competitive area and thus the support of $\nabla E$ becomes greater.

is a parabolic partial differential equation modelling a diffusion process. A parabolic equation has the nice property that information is spread with infinite speed and we can hope that this results in an improved convergence rate in contrast to non-parabolic evolution equations, such as (3). However, it might be a good idea, to limit the amount of information, which is spread across edges. Thus we will investigate not only the isotropic case $g \equiv 1$, but also

$$
g(x)=\frac{1}{1+|\nabla I(x)|^{2} / \beta},
$$

which is clearly anisotropic. Another consequence of (12) is that adding the proposed regulariser (9) makes the energy to be minimized more and more convex as $\lambda$ in (13) tends to infinity. Thus more and more local minima of $E$ to disappear. However, by choosing moderate values for $\lambda$, which means $\lambda \in[0.01,0.2]$, we will only discard very local and undesired minima.

\subsection{Practical Considerations}

Introducing a diffusion regularisation we cannot expect $\phi$ to be a signed distance function, even if it is initialized as such. Of course, this is also true for many other regularisation strategies and since only the zero level set of $\phi$ is interesting for level set segmentation, one is tempted not to worry too much about the shape of $\phi$. On the contrary, according to Gomes and Faugeras $[\Theta]$, an accurate approximation of derivatives of $\phi$ by finite differences is only guaranteed, if $\phi$ is not too steep. Also Chan and Vese [ब] as well as Li et al. [ $\square$ ] state that it is necessary to prevent $\phi$ from getting too steep or flat. As a consequence we want to make some effort to explain why $\phi$ cannot get too steep or flat in the case of diffusion regularisation. Therefore, we consider a typical energy, such as

$$
E(\phi)=\int_{\Omega} H(-\phi)\left(I-\mu_{i}\right)^{2}+H(\phi)\left(I-\mu_{o}\right)^{2} d x+\gamma \int_{\Omega} \delta(\phi) g(I)|\nabla \phi| d x,
$$

which has been proposed by Chan and Vese in [छ]. We further assume that $g(I) \equiv 1$ (w.l.o.g.), which leads to the diffusion equation

$$
\partial_{t} \phi=\delta(\phi)\left[\left(I-\mu_{i}\right)^{2}-\left(I-\mu_{o}\right)^{2}+\gamma \operatorname{div}\left(g \frac{\nabla \phi}{|\nabla \phi|}\right)\right]+\lambda \Delta \phi,
$$

where we assume Neumann boundary conditions at the borders of the image domain. 


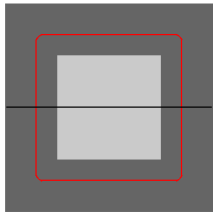

(a) initialisation

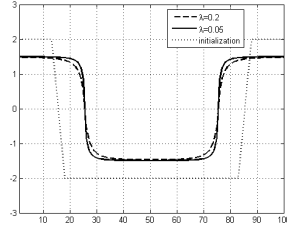

(b) $1 \mathrm{D}$ cut of the stationary state

Figure 2: The steepness of $\phi$ clearly depends on the choice of $\lambda$, but the position of the zero level set remains the same.

At first, we note that the numerical approximation of $\nabla E$ can be written in the form

$$
\nabla E(\phi(x))=\delta_{\varepsilon}(\phi(x)) F(x, \phi(x), I(x)),
$$

where

$$
\delta_{\varepsilon}(\phi)= \begin{cases}0, & |\phi|>\varepsilon, \\ \frac{1}{2 \varepsilon}\left(1+\cos \left(\frac{\pi \phi}{\varepsilon}\right)\right), & |\phi| \leq \varepsilon,\end{cases}
$$

is a smeared-out approximation of the Dirac delta distribution (see [ $\square$, Chapter 1] for further details) controlling the support of $\nabla E(\phi)$ and dividing the image domain into two areas:

- In the diffusion dominated area (indicated by grey stripes in Fig. 1), which corresponds to $\delta_{\varepsilon}(\phi)=0$, the evolution is completely governed by $\Delta \phi$. As a consequence and thanks to the Neumann boundary conditions we will observe that in the diffusiondominated area

$$
|\phi| \rightarrow \varepsilon \text { as } t \rightarrow \infty .
$$

- In the competitive area (indicated by grey colour in Fig. 1), which corresponds to $\delta_{\varepsilon}(\phi) \neq 0$, the evolution is governed by the competition between $\Delta \phi$ and $\nabla E(\phi)$. If $\phi$ becomes flatter, the competitive area and thus the support of $\nabla E$ increases as visualized in Fig. 1. At some point the increasing contribution of $\nabla E$ exceeds the one of $\Delta \phi$ causing $\phi$ to become steeper again. Conversely, if $\phi$ becomes flatter, the support and thus the influence of $\nabla E$ becomes smaller and at some point $\Delta \phi$ dominates causing $\phi$ to become flatter again. As a consequence, $\phi$ can neither become too flat, nor too steep in the competitive area. Indeed, by adjusting the regularisation parameter $\lambda$ we can even control how steep or flat $\phi$ becomes.

We conclude with a first numerical experiment demonstrating that the above made considerations can also be observed in practise. Thus we aim at segmenting the square in Fig. 2(a) and solve (16) by a semi-implicit scheme (c.f. Sec. 3). Initialized as a basin of depth 4, $\phi$ evolves smoothly towards a stationary configuration. We can observe in Fig. 2(b) that (19) holds true $(\varepsilon=1.5)$ and that the steepness of $\phi$ in the competitive area depends on $\lambda$.

\section{Efficient Numerical Schemes}

The direct translation of the gradient descent procedure for solving (6) results in the forward Euler scheme

$$
\phi^{t+\tau}=\phi^{t}-\tau \nabla E\left(\phi^{t}\right)+\tau \lambda \operatorname{div}\left(g \nabla \phi^{t}\right),
$$


where $\phi^{t}$ denotes the approximation of $\phi$ at time $t$. Provided powerful alternatives are available, which is the case here, one should not use a forward Euler scheme, because, even in the simplest case $g(I) \equiv 1$, it suffers from the severe CFL-condition $\tau \leq 1 / 4$ (in two spatial dimensions). Thus we now derive more efficient numerical schemes for solving (13).

\subsection{Semi-Implicit Discretisation}

In order to avoid severe time step constraints we can use the semi-implicit scheme

$$
\phi^{t+\tau}=\phi^{t}-\tau \nabla E\left(\phi^{t}\right)+\tau \lambda \operatorname{div}\left(g \nabla \phi^{t+\tau}\right),
$$

which leads to the update equation

$$
\phi^{t+\tau}=(I-\tau \lambda \operatorname{div}(g \nabla))^{-1}\left(\phi^{t}-\tau \nabla E\left(\phi^{t}\right)\right) .
$$

As the finite difference approximation of $I-\tau \lambda \operatorname{div}(g \nabla)$ is linear one can use efficient nu-

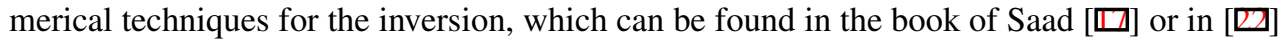
by Weickert et al.

\subsection{Duhamel's Principle}

If $g \equiv 1$, there is another possibility for solving (13) based on Duhamel's principle [ $\square$, Chapter 2]. The main idea is to split an initial value problem of the form

$$
\left\{\begin{array}{l}
\partial_{t} u(x, t)=f(x, t)+\lambda \Delta u(x, t) \\
u(x, 0)=g(x)
\end{array}\right.
$$

into two problems. One with the correct initial condition, but without source-term

$$
(i)\left\{\begin{array}{l}
\partial_{t} u_{i}(x, t)=\lambda \Delta u_{i}(x, t) \\
u_{i}(x, 0)=g(x)
\end{array}\right.
$$

and one including the source term, but with homogeneous initial condition

$$
(s)\left\{\begin{array}{l}
\partial_{t} u_{s}(x, t)=\lambda \Delta u_{s}(x, t)+f(x, t), \\
u_{s}(x, 0)=0
\end{array}\right.
$$

The superposition $u=u_{i}+u_{s}$ eventually solves the whole problem, because

$$
\partial_{t} u(x, t)=\left[\partial_{t} u_{i}+\partial_{t} u_{s}\right](x, t)=\left[\lambda \Delta u_{i}+\lambda \Delta u_{s}+f\right](x, t)=f(x, t)+\lambda \Delta u(x, t)
$$

and

$$
u(x, 0)=u_{i}(x, 0)+u_{s}(x, 0)=g(x)+0=g(x) .
$$

Denoting the fundamental solution by

$$
\Phi(x, t)= \begin{cases}(4 \pi \lambda t)^{-\frac{d}{2}} \exp \left(-\frac{\|x\|_{2}^{2}}{4 \lambda t}\right), & t>0 \\ 0, & t \leq 0\end{cases}
$$


where $d$ denotes the dimension, the solution to (i) can be written as

$$
u_{i}(x, t)=(\Phi * g)(x, t)
$$

if $t>0$. The solution to (s) can be obtained using Duhamel's principle:

$$
u_{s}(x, t)=\int_{0}^{t} \int_{\Omega} \Phi(x-y, t-s) f(y, s) d y d s .
$$

Approximating the integral with respect to $s$ we obtain

$$
u_{s}(x, t) \approx t \cdot \int_{\Omega} \Phi(x-y, t-0) f(y, 0) d y=t \cdot(\Phi * f)(x, 0),
$$

if $t>0$. Thus we can construct an iterative scheme for (13) as follows. At $t$ we take $\phi^{t}$ as the initial condition $g$ and $-\nabla E\left(\phi^{t}\right)$ is the corresponding source term $f$ and compute $\phi^{t+\tau}$ as

$$
\begin{aligned}
\phi^{t+\tau} & =G_{2 \sqrt{\tau \lambda}} * \phi^{t}-\tau \cdot G_{2 \sqrt{\tau \lambda}} * \nabla E\left(\phi^{t}\right) \\
& =G_{2 \sqrt{\tau \lambda}} *\left(\phi^{t}-\tau \cdot \nabla E\left(\phi^{t}\right)\right)
\end{aligned}
$$

where

$$
G_{2 \sqrt{\tau \lambda}}(x)=\Phi(x, 2 \sqrt{\tau \lambda})
$$

is nothing else than a Gaussian kernel with standard deviation $2 \sqrt{\tau \lambda}$.

\section{Regularisation Paradigms}

Now we want to relate the schemes derived in the last section with other ones from different regularisation strategies. In order to simplify the following considerations, we define three regularisation operators, which can be considered as low-pass filters or smoothing operators: the Gaussian operator

$$
\mathscr{G}(\sigma)[\psi]=G_{\sigma} * \psi
$$

the isotropic Sobolev operator

$$
\mathscr{S}(\alpha)[\psi]=(I-\alpha \Delta)^{-1} \psi
$$

and the anisotropic Sobolev operator

$$
\mathscr{A}(\alpha)[\psi]=(I-\alpha \operatorname{div}(g \nabla))^{-1} \psi .
$$

This allows to rewrite the derived schemes as follows:

$$
\phi^{t+\tau}= \begin{cases}\mathscr{G}(2 \sqrt{\tau \sigma})\left[\phi^{t}-\tau \cdot \nabla E\left(\phi^{t}\right)\right], & \text { Duhamel's principle for } g \equiv 1, \\ \mathscr{S}(\tau \lambda)\left[\phi^{t}-\tau \cdot \nabla E\left(\phi^{t}\right)\right], & \text { semi-implicit scheme for } g \equiv 1, \\ \mathscr{A}(\tau \lambda)\left[\phi^{t}-\tau \cdot \nabla E\left(\phi^{t}\right)\right], & \text { semi-implicit scheme for general } g\end{cases}
$$

If we forget about the model we want to solve and consider these schemes as plain update equations, we might wonder, whether it would be possible to regularize only the update 


\begin{tabular}{ccc}
\hline & applied to $\nabla E\left(\phi^{t}\right)$ & applied to $\phi^{t}-\tau \nabla E\left(\phi^{t}\right)$ \\
\hline $\mathscr{G}(\sigma)$ & {$[\mathbf{\theta}]$} & isotropic diffusion $(\sigma=2 \sqrt{\lambda \tau})$ \\
\hline $\mathscr{S}(\alpha)$ & {$[$ 目] and [四] } & isotropic diffusion $(\alpha=\lambda \tau)$ \\
\hline $\mathscr{A}(\alpha)$ & Weighted Sobolev Space & anisotropic diffusion $(\alpha=\lambda \tau)$ \\
\hline
\end{tabular}

Table 1: Overview over all possible update rules and the resulting models.

$\nabla E\left(\phi^{t}\right)$. Indeed, it is and this leads us to the implicit regularisation strategies suggested by Charpiat et al. [0] and Sundaramoorthi et al. [四]:

$$
\phi^{t+\tau}= \begin{cases}\phi^{t}-\tau \cdot \mathscr{G}(\sigma)\left[\nabla E\left(\phi^{t}\right)\right], & V=C^{\infty} \text { as in [四], } \\ \phi^{t}-\tau \cdot \mathscr{S}(\alpha)\left[\nabla E\left(\phi^{t}\right)\right], & V=H^{1} \text { as in [四] and [四]. }\end{cases}
$$

Obviously, one combination is missing here: it is

$$
\phi^{t+\tau}=\phi^{t}-\tau \cdot \mathscr{A}(\alpha)\left[\nabla E\left(\phi^{t}\right)\right], \quad V=H^{1},
$$

which corresponds to an implicit regularisation strategy based on the Sobolev space $H^{1}$, but equipped with a different scalar product:

$$
\langle\phi, \psi\rangle_{H^{1}}=\langle\phi, \psi\rangle_{L^{2}}+\alpha\langle\nabla \phi, g \nabla \psi\rangle_{L^{2}} .
$$

It is interesting that (40) has not been used for variational level set segmentation so far.

In summary, we can observe two regularisation paradigms here. We can apply a regularisation operator either to the whole right-hand side (rhs) $\phi^{t}-\tau \cdot \nabla E\left(\phi^{t}\right)$, or only to the update $\nabla E\left(\phi^{t}\right)$. The computational complexity is exactly the same in both cases. Of course, a convolution is faster to compute than the inversion of an equation system, but we can also speed up the application of $\mathscr{S}(\sigma)$, if we compute its impulse response $\mathscr{S}(\alpha) \delta$ and use it as a filter mask. This is, of course, not possible for $\mathscr{A}(\alpha)$. However, also $\mathscr{A}(\alpha)$ has its raison d'êtere as we will see in the next section. We conclude by referring to the overview presented in Tab. 1.

\section{Discussion of Numerical Experiments}

In order to compare the two regularisation paradigms derived in the last section, we segmented three images from [ $⿴ 囗 \mathbf{0}$ (see Fig. 3) based on the energy defined in (15). We used $\tau=10$ and the following parameter settings regarding the energy to be minimised: $\lambda=0.1$ in Eq. (13) as well as $\gamma=0.2$ (plane), $\gamma=0.5$ (moth), $\gamma=0.1$ (leaf) in Eqn. (15). In order to keep the application of the regularisation operators corresponding to the implicit strategies of $[\mathbf{\square}, \mathbf{Q}, \mathbf{⿴ 囗 十}]$ comparable we used $\sigma=2 \sqrt{\tau \lambda}=2$ and $\alpha=\tau \lambda=1$ in Eqn. (39) and Eqn. (40).

A general observation is that it is always advisable to apply a regularisation operator to the whole right-hand side and not only to the update, which corresponds to a diffusion regularisation. In all three cases this results in an increased convergence rate (c.f. Fig. 3(c), 3(f), and 3(i)) and a smoother embedding function. Moreover, if we compare the results in Fig. 4, the regularisation of the whole right hand side seems to be less prone to get stuck in a local minimum of $E$. 


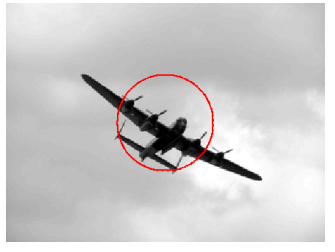

(a) initialisation

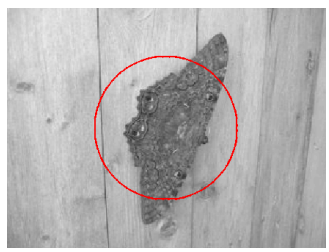

(d) initialisation

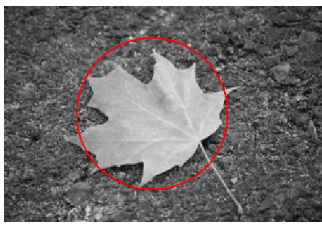

(g) initialisation

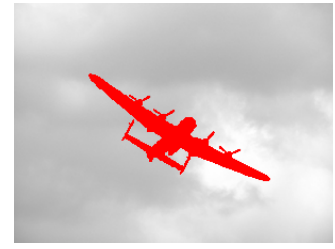

(b) ground truth

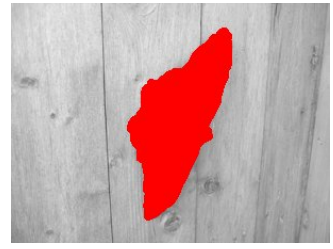

(e) ground truth

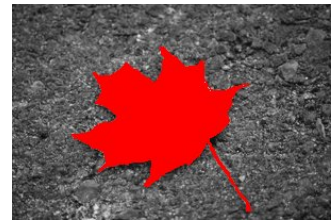

(h) ground truth

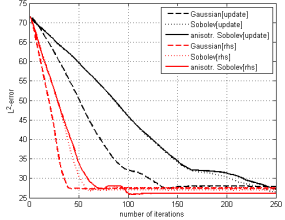

(c) convergence rates

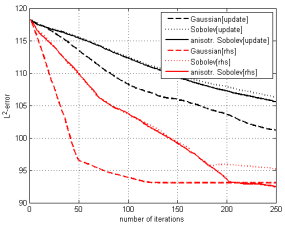

(f) convergence rates

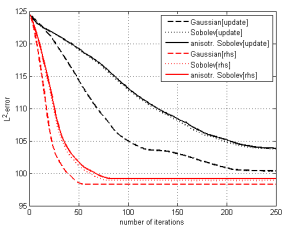

(i) convergence rates

Figure 3: The diffusion-based regularisation paradigm shows an increased convergence rate.

Comparing the three regularisation operators with each other, it turns out that the Sobolev operator $\mathscr{S}(\alpha)$ enjoys the best compromise between runtime and quality: on the one hand, it can be implemented via a convolution with its impulse response $\mathscr{S}(\alpha) \delta$, which allows for a short runtime, and on the other hand, the quality of the results is visually satisfying.

\section{References}

[1] S. Alpert, M. Galun, R. Basri, and A. Brandt. Image segmentation by probabilistic bottom-up aggregation and cue integration. In Computer Vision and Pattern Recognition, 2007. CVPR '07. IEEE Conference on, pages 1 -8, 17-22 2007. doi: 10.1109/CVPR.2007.383017.

[2] Leah Bar and Guillermo Sapiro. Generalized newton-type methods for energy formulations in image processing. SIAM Journal on Imaging Sciences, 2(2):508-531.

[3] O. Bernard, D. Friboulet, P. Thevenaz, and M. Unser. Variational b-spline level-set: A linear filtering approach for fast deformable model evolution. Image Processing, IEEE Transactions on, 18(6):1179 -1191, june 2009. ISSN 1057-7149. doi: 10.1109/TIP. 2009.2017343 .

[4] Xavier Bresson, Selim Esedoglu, Pierre Vandergheynst, Jean-Philippe Thiran, and Stanley Osher. Fast global minimization of the active contour/snake model. Journal of Mathematical Imaging and Vision, 28. 


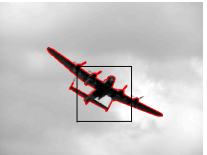

(a) $\mathscr{G}$ update

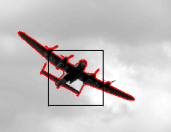

(b) $\mathscr{S}$ update

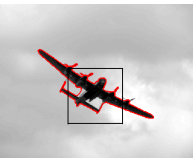

(c) $\mathscr{A}$ update

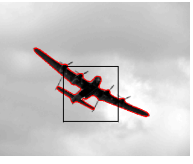

(d) $\mathscr{G}$ rhs

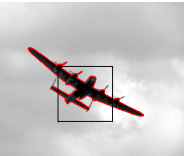

(e) $\mathscr{S}$ rhs

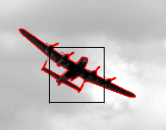

(f) $\mathscr{A}$ rhs
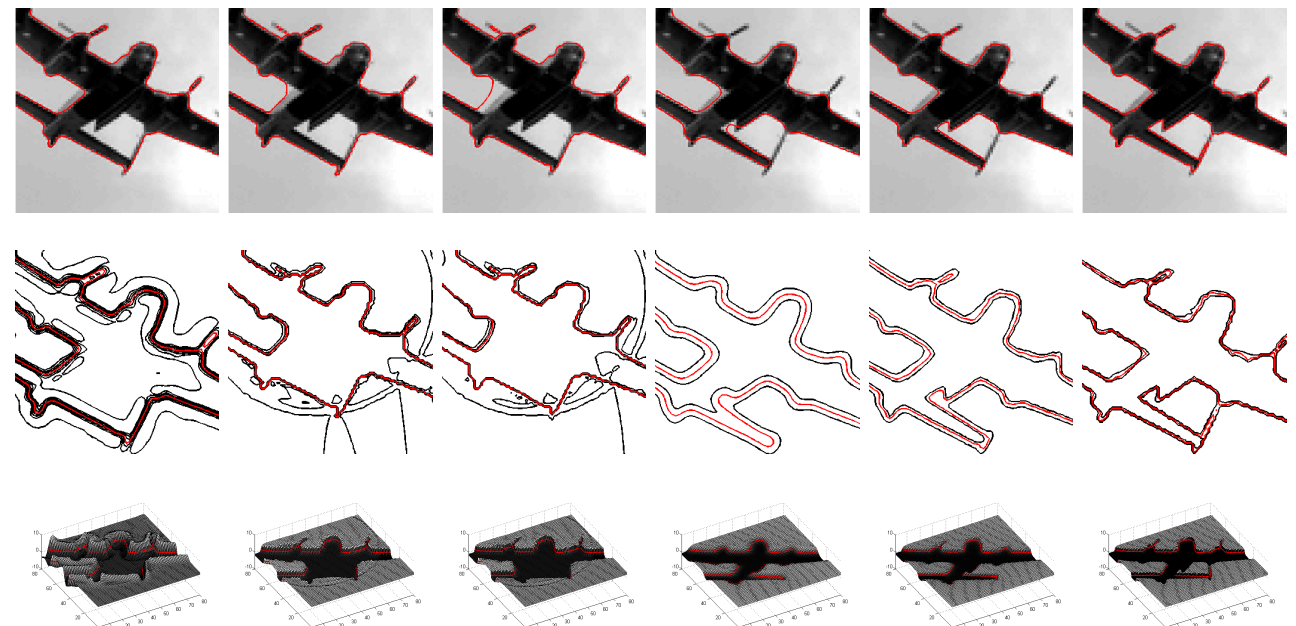

Figure 4: Segmentation results (first row), close-ups of the results (second row), the corresponding contour plots (third row), and the corresponding surface plots (bottom row).

[5] T.F. Chan and L.A. Vese. Active contours without edges. Image Processing, IEEE Transactions on, 10(2):266-277, Feb 2001.

[6] G. Charpiat, P. Maurel, J.-P. Pons, R. Keriven, and O. Faugeras. Generalized gradients: Priors on minimization flows. Int. J. Comput. Vision, 73(3):325-344, 2007.

[7] Lawrence C. Evans. Partial differential equations, volume 19 of Graduate Studies in Mathematics. American Mathematical Society, 1998.

[8] José Gomes and Olivier D. Faugeras. Reconciling distance functions and level sets. In SCALE-SPACE '99: Proceedings of the Second International Conference on ScaleSpace Theories in Computer Vision, pages 70-81, 1999.

[9] Hon Pong Ho, Yunmei Chen, Huafeng Liu, and Pengcheng Shi. Level set active contours on unstructured point cloud. In Computer Vision and Pattern Recognition, 2005. CVPR 2005. IEEE Computer Society Conference on, volume 2, pages 655 - 662 vol. 2, 20-25 2005. doi: 10.1109/CVPR.2005.211.

[10] Xiaolei Huang, D. Metaxas, and Ting Chen. Metamorphs: Deformable shape and texture models. In Computer Vision and Pattern Recognition, 2004. CVPR 2004. Proceedings of the 2004 IEEE Computer Society Conference on, volume 1, pages I-496 I-503 Vol.1, 27 2004. doi: 10.1109/CVPR.2004.1315072.

[11] Chunming Li, Chenyang Xu, Changfeng Gui, and M.D. Fox. Level set evolution without re-initialization: a new variational formulation. In Computer Vision and Pattern 
Recognition, 2005. CVPR 2005. IEEE Computer Society Conference on, volume 1, pages 430 - 436 vol. 1, 20-25 2005. doi: 10.1109/CVPR.2005.213.

[12] R. Malladi, J.A. Sethian, and B.C. Vemuri. Shape modeling with front propagation: a level set approach. Pattern Analysis and Machine Intelligence, IEEE Transactions on, 17(2):158 -175, feb 1995.

[13] B.S. Morse, Weiming Liu, T.S. Yoo, and K. Subramanian. Active contours using a constraint-based implicit representation. In Computer Vision and Pattern Recognition, 2005. CVPR 2005. IEEE Computer Society Conference on, volume 1, pages 285 - 292 vol. 1, 20-25 2005. doi: 10.1109/CVPR.2005.59.

[14] Benoit Mory, Roberto Ardon, Anthony J. Yezzi, and Jean-Philippe Thiran. Noneuclidean image-adaptive radial basis functions for $3 \mathrm{~d}$ interactive segmentation. In Computer Vision, 2009 IEEE 12th International Conference on, pages 787 -794, sept. 2009. doi: 10.1109/ICCV.2009.5459245.

[15] David Mumford and Jayant Shah. Optimal approximations by piecewise smooth functions and associated variational problems. Communications on Pure and Applied Mathematics, 42(5):577-685, 1989.

[16] Stanley Osher and Ronald Fedkiw. Level Set Methods and Dynamic Implicit Surfaces. Springer, 2003.

[17] Y. Saad. Iterative Methods for Sparse Linear Systems. Society for Industrial and Applied Mathematics, Philadelphia, PA, USA, 2003. ISBN 0898715342.

[18] Christoph SchnÃûrr. A study of a convex variational diffusion approach for image segmentation and feature extraction. J. Math. Imaging Vision, 8:271-292, 1998.

[19] G. Slabaugh, Quynh Dinh, and G. Unal. A variational approach to the evolution of radial basis functions for image segmentation. pages $1-8,17-22$ 2007. doi: 10.1109/ CVPR.2007.383013.

[20] Ganesh Sundaramoorthi, Anthony J. Yezzi, and Andrea Mennucci. Sobolev active contours. International Journal of Computer Vision, 73(3):345-366, 2007.

[21] Alain Trouvé. Diffeomorphisms groups and pattern matching in image analysis. International Journal of Computer Vision, 28(3):213-221.

[22] J. Weickert, B.M.T.H. Romeny, and M.A. Viergever. Efficient and reliable schemes for nonlinear diffusion filtering. Image Processing, IEEE Transactions on, 7(3):398 -410, 1998. 